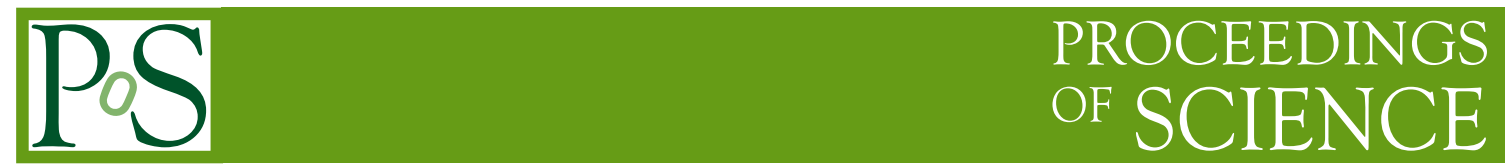

\title{
One- and two-loop integrals with XLOOPS-GiNaC
}

\author{
H.S. Do ${ }^{* a}$ Phan Hong Khiem ${ }^{b}$ and F. Yuasa ${ }^{c}$ \\ ${ }^{a}$ College of Science, Vietnam National University, \\ 227 Nguyen Van Cu, Dist.5, Ho Chi Minh City, Vietnam \\ ${ }^{b}$ Thu Dau Mot University, \\ O6 Tran Van On, Thu Dau Mot Town, Binh Duong Province, Vietnam \\ ${ }^{c}$ High Energy Accelerator research Organization (KEK), \\ 1-1 OHO Tsukuba, Ibaraki 305-0801, Japan \\ E-mail: dhson@hcmus.edu.vn, khiemph@tdm.edu.vn, fukuko.yuasa@kek.jp
}

In this paper, we report the progress of XLOOPS-GiNaC [1] in one-and two-loop computations. Explicit results of scalar one-loop box diagram are shown in comparison with LoopTools' [2, 3] and of the Direct Computation Method [4].

CPP2010, 3rd Computational Particle Physics Workshop KEK (Tsukuba), Japan

September, 23rd-25th 2010

*Speaker. 


\section{Introduction}

To regularize the Landau singularity in the next-to-leading order calculations for processes at LHC and future ILC, loop diagrams with complex massive particles are interested.

In this paper, we present the progress in the development of XLOOPS-GiNaC and show the results of Feynman scalar one-loop box integral with complex masses. The loop integration is performed directly in parallel and orthogonal space (POS) of internal momentum following the method by Kreimer [5]. The method possibly opens a new way to avoid the singularity of the Gram determinant and to regularize Landau poles simultaneously.

\section{Progress in development of XLOOPS-GiNaC}

The aim of XLOOPS-GiNaC is to provide a C++ library to calculate one- and two-loop one, two, three, and four-point Feynman integrals with arbitrary masses (zero, real or complex masses) and at any tensor rank. It is based on GiNaC [6], a CAS written in C++.

The structure of XLOOPS-GiNaC is shown in the Fig. 1. XLOOPS-GiNaC provides a com-
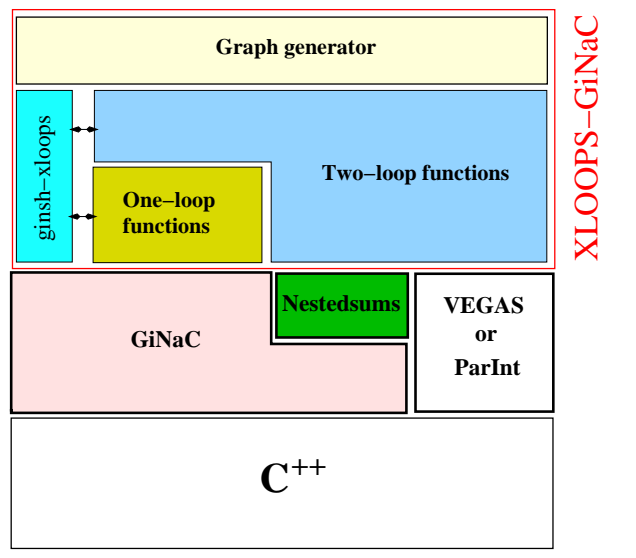

Figure 1: The structure of XLOOPS-GiNaC

plete C++ library for both one- and two-loop functions, and a simple interactive interface via ginsh-xloops for one-loop functions. For numerical integration of two-loop functions, VEGAS [7] or ParInt package [8] is used. The analytical part of two-loop functions is written in terms of Hypergeometric functions that can be evaluated by nestedsum library [9].

At the current state, the one-loop one-, two-, and three-point functions are tested and implemented into XLOOPS-GiNaC. The two-loop two-point and four-point functions are not complete and still in implementation and testing phase.

In this paper, we introduce a new function for the scalar one-loop four-point integral with complex masses into XLOOPS-GiNaC. Details of the calculation will be published elsewhere [10]. The algorithm for the new function is actually an extension of the previous works done by Franzkowski for scalar one-loop four-point integrals with real massive particles [11]. Different to the previous version of XLOOPS-GiNaC where the input parameters are written in parallel and orthogonal 
components of momenta, the new function uses the de facto input parameters of LoopsTools. The syntax of the new function is as follow

$$
\text { Scalar_OneLoop_4Pt(p1, p2, p3,p4, p1p2, p2p3, m1, m2, m3, m4, rho); }
$$

where the scalar one-loop four-point integral is defined by

$$
\int d^{4} l \frac{1}{\prod_{i=1}^{4}\left[\left(l+q_{i}\right)^{2}-m_{i}^{2}+i \rho\right]}
$$

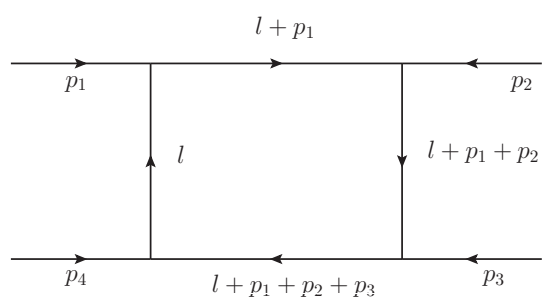

with $p 1 p 2=\left(p_{1}+p_{2}\right)^{2}, p 2 p 3=\left(p_{2}+p_{3}\right)^{2}$, and $q_{i}=\sum_{j=1}^{i} p_{j}$. The masses can be complex $m_{i}^{2}=$ $m_{0 i}^{2}-i m_{0 i} \Gamma_{i}$. The width of unstable particles, $\Gamma_{i}$, is positive.

Because of changing to the new interface, one-loop four-point function has been temporary implemented in a separate module and will be merged into XLOOPS-GiNaC when the interface of all the other one-loop functions (one-, two-, and three-point) are rewritten.

\section{The results}

In this section, we present some results of the new one-loop four-point module which can be compared to that of LoopTools 2.5 and of Direct Computation Method.

\subsection{Scalar one-loop box diagram}

We consider one-loop box diagram with complex masses which contributes to the process $g g \rightarrow b \bar{b} H$ as in Fig. 2. To compare with the results of [12] we chose the input parameters for XLOOPS-GiNaC as follow: $p_{5}^{2}=M_{H}^{2}=27225 \mathrm{GeV}^{2}, p_{3}^{2}=p_{4}^{2}=M_{b}^{2}=0 \mathrm{GeV}^{2}, s^{2}=\left(p_{1}+p_{2}\right)^{2}=$ $124609 \mathrm{GeV}^{2}, M_{0 t}=174.0 \mathrm{GeV}, \Gamma_{t}=1.5 \mathrm{GeV}, M_{0 W}=80.3766 \mathrm{GeV}, \Gamma_{W}=2.1 \mathrm{GeV}, \sqrt{s_{1}}=$ $271.06 \mathrm{GeV}$

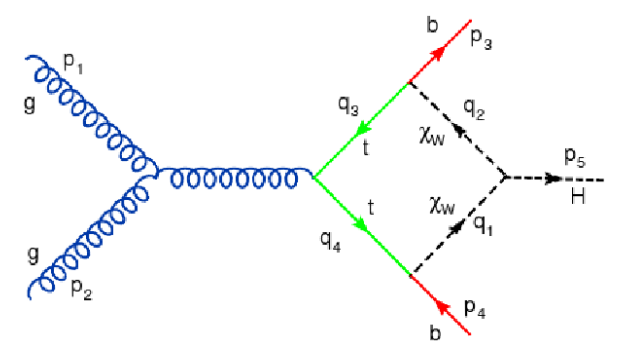

Figure 2: The one-loop box diagram of process $g g \rightarrow b \bar{b} H$ from [12]

The real and imaginary parts of the scalar one-loop box integral which are calculated by XLOOPS-GiNaC is shown in Fig. 3. In the real masses case, a Landau singularity appears at 

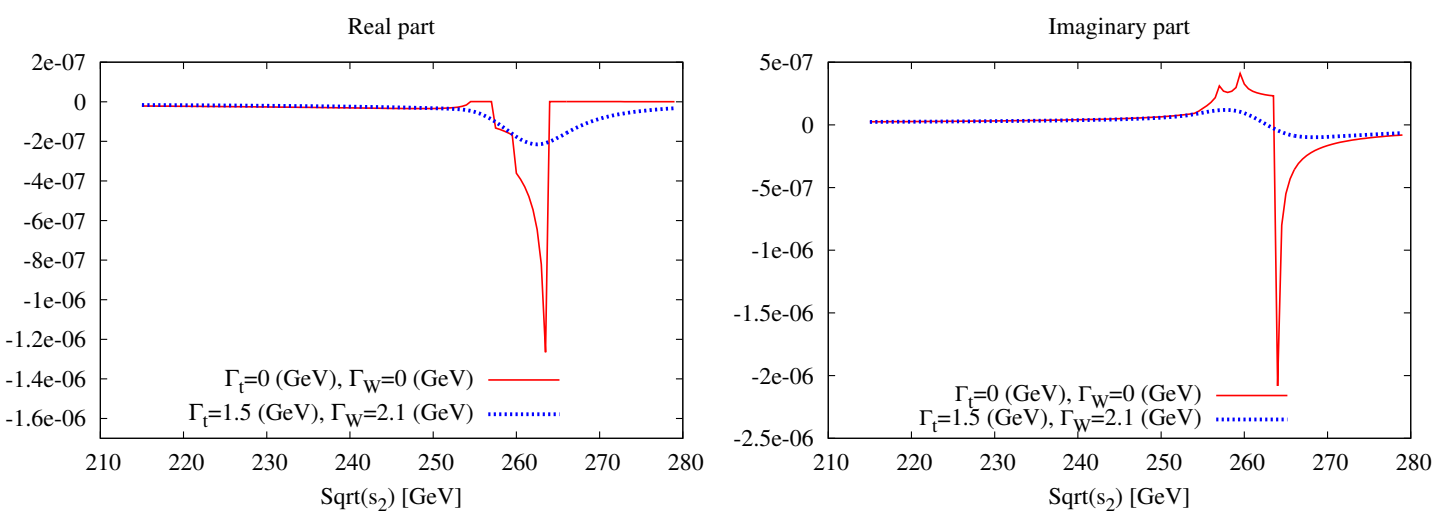

Figure 3: The real and the imaginary part of the scalar one-loop four-point integral that contributes to $g g \rightarrow b \bar{b} H$

$\sqrt{s_{2}}=\sqrt{\left(p_{4}+p_{5}\right)^{2}}=264 \mathrm{GeV}$. When the widths $\Gamma_{t}=1.5 \mathrm{GeV}$ and $\Gamma_{W}=2.1 \mathrm{GeV}$ are inserted into the masses, Landau pole is regularized. These results are in good agreement with [12].

In Table 1 we show the numerical results of XLOOPS-GiNaC along with that of LoopTools 2.5 and of Direct Computation Method at some points in the configuration space. The computing speed is as fast as of LoopTools. In Table 2, other comparisons with LoopTools in various configurations of masses and momenta including timelike, spacelike and lightlike cases are shown. All the results are in good agreement with LoopTools.

\begin{tabular}{ccccc}
\hline$m_{3}^{2}$ & real/imag. & XLOOPS-GiNaC & LoopTools 2.5 & Direct Computaion Method \\
\hline \hline 10 & Real & $2.476607718585036053 E^{-4}$ & 0.000247661 & $0.2476607718651927 E^{-03}$ \\
& Imag. & $4.830551285215682584 E^{-4}$ & 0.000483055 & $0.4830551284955444 E^{-03}$ \\
\hline \multirow{2}{*}{100} & Real & $8.1543415594642731853 E^{-5}$ & $8.15434 E^{-05}$ & $0.8154341560206131 E^{-04}$ \\
& Imag. & $1.5450057163059433522 E^{-4}$ & 0.000154501 & $0.1545005716281967 E^{-03}$ \\
\hline 1000 & Real & $1.47691716458090845525 E^{-5}$ & $1.47692 E^{-05}$ & $0.1476917164636933 E^{-04}$ \\
& Imag. & $2.2197908354423311047 E^{-5}$ & $2.21979 E^{-05}$ & $0.2219790835451625 E^{-04}$ \\
\hline
\end{tabular}

Table 1: Input parameters (in $\mathrm{GeV}^{2}$ ) used in the calculations: $p_{1}^{2}=10, p_{2}^{2}=-60, p_{3}^{2}=-10, p_{4}^{2}=-10$, $\left(p_{1}+p_{2}\right)^{2}=200,\left(p_{2}+p_{3}\right)^{2}=-10, m_{1}^{2}=10-5 i, m_{2}^{2}=20-0 i, m_{4}^{2}=40-10 i$.

\subsection{Toward two-loop box diagrams}

The POS method was applied to evaluate UV-finite two-loop box diagrams with real masses [13]. Recently, we continue to extend the works of R. Kreckel et al to a same set of integrals including complex masses cases. After careful investigation the structure of two-loop integrals, we noticed that the same integration procedure of scalar one-loop box integral can be used to reduce eight-fold integrals of scalar two-loop box diagrams down to four-folds integrals those are inputs of numerical integration step. The interested diagrams are shown in Fig. 4. However, the stability of numerical integration is the biggest issue that we have to tame at the moment. 


\begin{tabular}{cccc}
\hline$\left(p_{1}^{2}, p_{2}^{2}, p_{3}^{2}, p_{4}^{2}, p 1 p 2, p 2 p 3\right)$ & Real/Imag. & XLOOPS-GiNaC & LoopTools 2.5 \\
\hline \hline$(10,60,10,90,200,10)$ & Real & $-7.6754958275901916 E^{-4}$ & -0.00076755 \\
& Imag. & $7.9369236308335912 E^{-4}$ & 0.000793692 \\
\hline$(10,60,-10,90,200,10)$ & Real & $-6.221361528827869 E^{-4}$ & -0.000622136 \\
& Imag. & $8.2028978832352737 E^{-4}$ & 0.00082029 \\
\hline$(10,60,-10,-10,200,-10)$ & Real & $-2.8547305408820357 E^{-4}$ & -0.000285473 \\
& Imag. & $5.894737433975371 E^{-4}$ & 0.000589474 \\
\hline$(10,-60,-10,-90,200,-10)$ & Real & $1.531845524366800 E^{-4}$ & 0.000153185 \\
& Imag. & $2.561867870169164 E^{-4}$ & 0.000256187 \\
\hline$(10,60,0,90,200,10)$ & Real & $-6.9233899095796788 E^{-4}$ & -0.000692339 \\
& Imag. & $8.1110140262125966 E^{-4}$ & 0.000811101 \\
\hline$(10,60,0,0,200,-10)$ & Real & $-3.3499315746623337 E^{-4}$ & -0.000334993 \\
& Imag. & $6.178621892720976 E^{-4}$ & 0.000617862 \\
\hline$(10,0,0,0,200,-10)$ & Real & $9.195393358045053 E^{-5}$ & $9.19539 E^{-05}$ \\
& Imag. & $5.2601566543985510 E^{-4}$ & 0.000526016 \\
\hline
\end{tabular}

Table 2: Input parameters (in $\mathrm{GeV}^{2}$ ) used in the calculations: $m_{1}^{2}=10-5 i, m_{2}^{2}=20-2 i, m_{3}^{2}=30-3 i$, $m_{4}^{2}=40-4 i$, and $\rho=10^{-30}$.
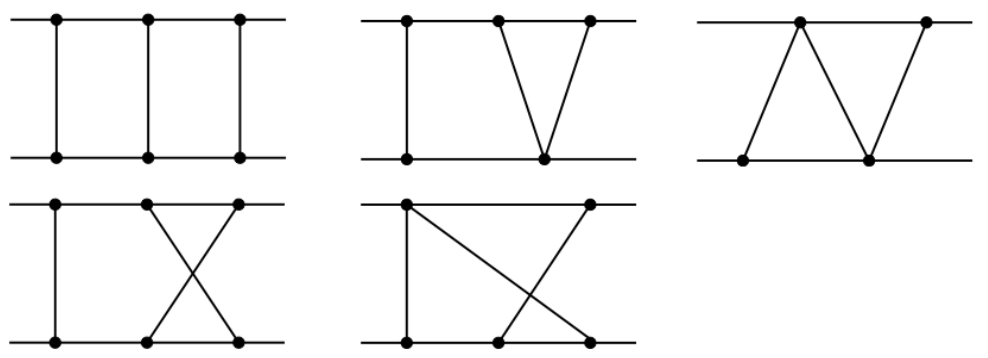

Figure 4: The two-loop box diagram [13]

\section{Conclusion}

In this paper, we presented various results of evaluating the scalar one-loop four-point integral. The results are carefully compared with known results of LoopTools 2.5 and Direct Computation Method. The results are in good agreement.

In the next step, we will extend the calculation procedure to evaluate tensor one-loop fourpoint integrals as well as two-loop box integrals. We hope to archive a computer program for one and two loop evaluation with Gram determinant singularity free.

\section{Acknowledgments}

We would like to thank P. Aurenche, F. Boudjema, Y. Kurihara, T. Kaneko, Le Duc Ninh and J. Vollinga for fruitful discussions. The research on scalar one-loop box carried out within the scope of the France-Vietnam Particle Physics Laboratory (FV-PPL LIA). This work was supported in part by the Exchange Program for East Asian Young Researchers of the Japan Society for the Promotion of Science (JSPS) 


\section{References}

[1] C. Bauer and H.S. Do, Comput. Phys. Commun. 144 (2002) [hep-ph/0102231];

L. Brücher, J. Franzkowski and D. Kreimer, Comput. Phys. Commun. 85 (1995);

Comput. Phys. Commun. 107 (1997);

L. Brücher, J. Franzkowski, A. Frink and D. Kreimer, Nucl. Instrum. Meth. A389 (1997).

[2] G.J. van Oldenborgh, Comput. Phys. Commun. 66 (1991).

[3] DAO Thi Nhung and LE Duc Ninh, Comput.Phys.Commun. 180 (2009).

[4] F. Yuasa et al., PoS (ACAT08) 122 (2008).

[5] D. Kreimer, Z.Physic. C54 (1992).

[6] C. Bauer, A. Frink and R. Kreckel, J. Symbolic Computation 33 (2002).

[7] G.P. Lepage, CLNS-80/447 (1980);

G.P. Lepage, J. Comp. Phys. 27 (1978);

R. Kreckel, [MZ-TH/97-30].

[8] L. Cucos and E. de Doncker, in proceedings of High Performance Computing Symposium 2002, POS (HPC02) 2002.

[9] S. Weinzierl, Comp. Phys. Comm. 145 (2002) [math-ph/0201011]

[10] H.S. Do and Phan Hong Khiem, to be published.

[11] J. Franzkowski, Dissertation, Mainz 1997.

[12] F. Boudjema and LE Duc Ninh, Phys. Rev. D78 (2008).

[13] R. Kreckel, D. Kreimer and K. Schilcher, Eur. Phys. J. C6 (1999). 